\title{
A systematic mRNA control mechanism for germline stem cell homeostasis and cell fate specification
}

\author{
Myon-Hee Lee ${ }^{1,4, *}$, Srivalli Swathi Mamillapalli ${ }^{1}$, Brett D. Keiper ${ }^{2}$ \& Dong Seok Cha ${ }^{3}$ \\ ${ }^{1}$ Department of Medicine, Hematology/Oncology Division, ${ }^{2}$ Department of Biochemistry and Molecular Biology, Brody School of Medicine, \\ East Carolina University, Greenville, NC 27834, USA, ${ }^{3}$ Department of Oriental Pharmacy, College of Pharmacy, Woosuk University, Jeonju \\ 55338, Korea, ${ }^{4}$ Lineberger Comprehensive Cancer Center, University of North Carolina-Chapel Hill, Chapel Hill, NC 27599, USA
}

\begin{abstract}
Germline stem cells (GSCs) are the best understood adult stem cell types in the nematode Caenorhabditis elegans, and have provided an important model system for studying stem cells and their cell fate in vivo, in mammals. In this review, we propose a mechanism that controls GSCs and their cell fate through selective activation, repression and mobilization of the specific mRNAs. This mechanism is acutely controlled by known signal transduction pathways (e.g., Notch signaling and Ras-ERK MAPK signaling pathways) and P granule (analogous to mammalian germ granule)-associated $\mathrm{mRNA}$ regulators (FBF-1, FBF-2, GLD-1, GLD-2, GLD-3, RNP-8 and IFE-1). Importantly, all regulators are highly conserved in many multi-cellular animals. Therefore, GSCs from a simple animal may provide broad insight into vertebrate stem cells (e.g., hematopoietic stem cells) and their cell fate specification. [BMB Reports 2016; 49(2): 93-98]
\end{abstract}

\section{C. elegans GERMLINE}

Germline stem cells (GSCs) are characterized by their ability to both self-renew and generate gametes - sperm or eggs. In the adult gonads of many organisms, GSCs are maintained to replenish stocks of germ cells whose numbers are depleted by gamete production. GSCs are also responsible for transmitting genetic information across the generations. A systematic regulatory network, including extrinsic cues and intrinsic regulation, tightly regulates a balance between self-renewal and differentiation of GSCs (called "GSC homeostasis") (1, 2). Therefore, aberrant regulation of this network can result in either loss of a specific germ cell type (arrested gametogenesis

*Corresponding author. Tel: +1(252)744-3134; Fax: +1(252)7443418; E-mail: leemy@ecu.edu

http://dx.doi.org/10.5483/BMBRep.2016.49.2.135

Received 30 June 2015, Revised 17 August 2015, Accepted 18 August 2015

Keywords: Germline stem cells, Cell fate specification, Caenorhabditis elegans, mRNA selection, Translational control resulting in sterility) or over-proliferation of undifferentiated germ cells, which are associated with germline tumors (3).

The nematode $C$. elegans is a very versatile reproductive model organism that has greatly contributed to the understanding of germline development (4). C. elegans exist as either hermaphrodites or males. Hermaphrodites produce a limited number of sperm in early larval stage (L3 to early L4) and switch to produce only oocytes in late larval stages (4). Thus, they are self-fertile (Fig. 1A). Infrequently occuring males produce sperm continuously without switching into oogenesis (Fig. 1B). Since hermaphrodites produce both sperm and oocytes from the same GSC, their germlines can be a good model system to study a mechanism for GSC homeostasis and cell fate specification. Moreover, the C. elegans germline is organized in a simple linear pattern that progresses from GSCs at the distal region to maturing gametes at the proximal region (Fig. 1A and 1B).

In the C. elegans gonad, a single mesenchymal somatic cell, called the distal tip cell (DTC), functions as a stem cell niche (also known as "microenvironment"), and drives the mitotic cell cycle in adjacent GSCs $(4,5)$ (Fig. 1C). Although specific individual GSC types have not been precisely defined in the $C$. elegans, genetic and cellular analyses suggest that GSCs are located in the distal mitotic region that directly contacts the DTC $(4,6)$ (Fig. 1C). As a GSC leaves the DTC niche, it enters the meiotic cell cycle and eventually differentiates into either sperm or oocytes (Fig. 1A and 1B). Notably, several RNA-binding proteins (e.g., PUF (Pumilio and FBF) (7)) control both GSC homeostasis and germ cell fate in the $C$. elegans germline. This observation suggests that regulatory mechanisms for the self-renewal/differentiation decision and sperm/oocyte decisions may be closely linked (8).

A comparative analysis of GSC regulatory mechanism in Drosophila, C. elegans, and mouse models has elucidated fundamental principles for self-renewal and differentiation (9). In these model systems, extrinsic factors (called niche signals) activate transcription for promoting the self-renewal of GSCs and preventing GSCs from differentiation. The extrinsic factors include Notch, Bone Morphogenic Protein (BMP) and Janus Kinase-Signal Transduction and Activator of Transcription (JAK/STAT) signaling pathways, more specific examples in- 
A
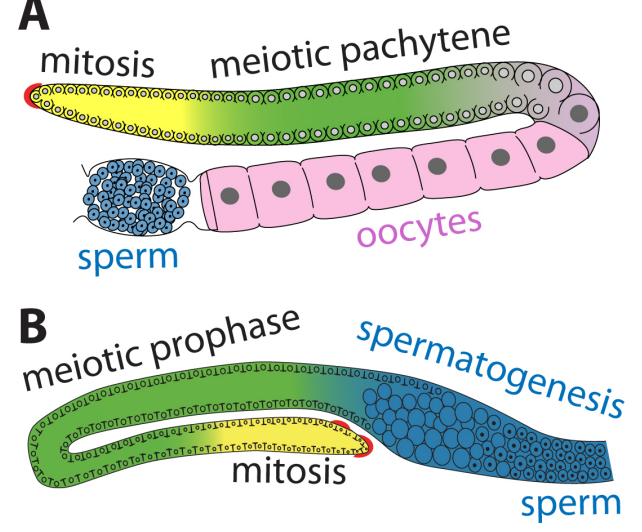

\section{C mitotic region/GSCs meiotic

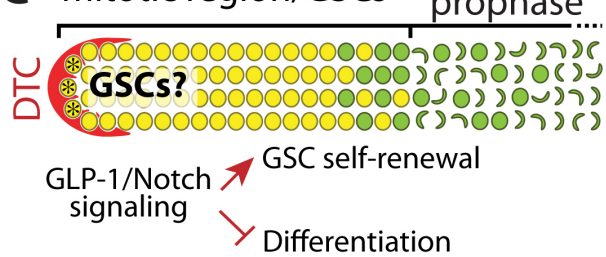

Fig. 1. C. elegans germline and GSC regulation. (A) Schematic of an adult C. elegans hermaphrodite gonad. Somatic DTC is located at the distal end. Cells at the distal end of the germline, including GSCs, divide mitotically (yellow). As cells move proximally, they enter meiosis (green) and differentiate into either sperm (blue) or oocytes (pink). (B) Schematic of an adult C. elegans male gonad. Two somatic DTCs reside at the distal ends of the adult male gonad. In the male germline, all GSCs differentiate into sperm (blue). (C) A simplified model for GSCs and their differentiation. GSC self-renew and differentiate into either sperm or egg. GLP-1/Notch signaling promotes GSC self-renewal and proliferation by inhibiting differentiation. *, inferred actual GSCs.

clude: Piwi/Yb in Drosophila, Notch signaling in C. elegans and Glial cell line-Derived Neutrophic Factor (GDNF) and possibly BMP signaling pathways in mouse testis (9).

In addition to these extrinsic factors several classes of intrinsic factors, including translational regulators (Pumilio, Nanos, Bam, SCF/c-kit, and Plzf), also control the self-renew$\mathrm{al} /$ differentiation of GSCs and cell lineage commitment (9). Although different combinations of extrinsic factors and intrinsic factors are need for GSC self-renewal and differentiation in different systems, a systematic mRNA control mechanism through an intimate interplay between extrinsic factors and intrinsic factors may be involved in controlling GSC homeostasis and cell fate specification.

In this review, we propose a mechanism to explain the control of GSC homeostasis and their cell fate specification through systematic and episodic mRNA selection. Most regulators identified in C. elegans are highly conserved in multi-cellular animals and have been implicated in stem cell con- trol and cell fate specification. The streamlined C. elegans gonads may provide a useful platform to understand mechanisms underlying stem cell regulation and cell fate specification in higher model systems, including humans.

\section{TRANSCRIPTIONAL ACTIVATION BY GLP-1/NOTCH SIGNALING IN GSCs}

In C. elegans, the DTC functions as a GSC niche and promotes the mitotic cell cycle in germ cells through a signal transduction pathway initiated by GLP-1 (one of two C. elegans Notch receptors) (3-5) (Fig. 2A). The GLP-1/Notch signal maintains the germ cells in the undifferentiated state through the transcriptional activation of target genes (10-12) (Fig. 2B). The Notch signaling pathway and its core components in C. elegans are highly conserved: the Notch ligand "LAG-2 (a family of DSL ligands)" is expressed in DTCs and its receptor, "GLP-1", is expressed on the membranes of mitotically dividing germ cells $(3,13)$ (Fig. 2A).

In the absence of signaling or progression of meiotic cell cycle, the transcription factor, "LAG-1 (a family of CSL transcription effectors)", is associated with a repressor complex to inhibit the expression of GLP-1/Notch target genes. Upon signal activation, an ADAM-family metalloprotease and $\gamma$-secretase cleaves the GLP-1/Notch receptor, and its intracellular domain (NICD) translocates from membrane to the nucleus. In the nucleus, NICD interacts with LAG-1 and LAG-3 (a homolog of mastermind transcriptional co-activators) to activate the expression of Notch target genes (Fig. 2A). Therefore, identifying the direct GLP-1/Notch target genes driving GSC self-renewal is crucial for understanding the molecular mechanisms of normal stem cell regulation, as well as tumorigenesis mediated by aberrant Notch signaling.

Recently, bioinformatics has identified 163 putative GLP-1/Notch target genes, all harboring clusters of at least four LAG-1 binding sites (LBSs) (14). Among them, four genes were validated as bona fide GLP-1/Notch targets in the C. elegans germline. These include FBF-2 (a family of PUF RNA-binding proteins) (15), LIP-1 (a homolog of the dual-specificity phosphatase) (16), SYGL-1 (SYnthetic GLp-1) (14) and LST-1 (Lateral Signaling Target-1) (14) (Fig. 2B). These genes function redundantly to maintain GSCs in C. elegans, while fbf-2, lip-1, sygl-1, and Ist-1 single mutants appear normal $(7,14-17)$, the Ist-1 sygl-1 double mutant very nearly pheno-copies the g/p-1 loss-of-function mutant, which is unable to maintain GSCs (14). Notably, the fbf-2; lip-1 double mutant displays a defect in germ cell fate specification, rather than GSC maintenance (Lee et. al., unpublished results) (Fig. 2C). These observations suggest that GLP-1/Notch signaling and its targets may regulate both GSC maintenance and germ cell fate specification in the C. elegans germline. 
A
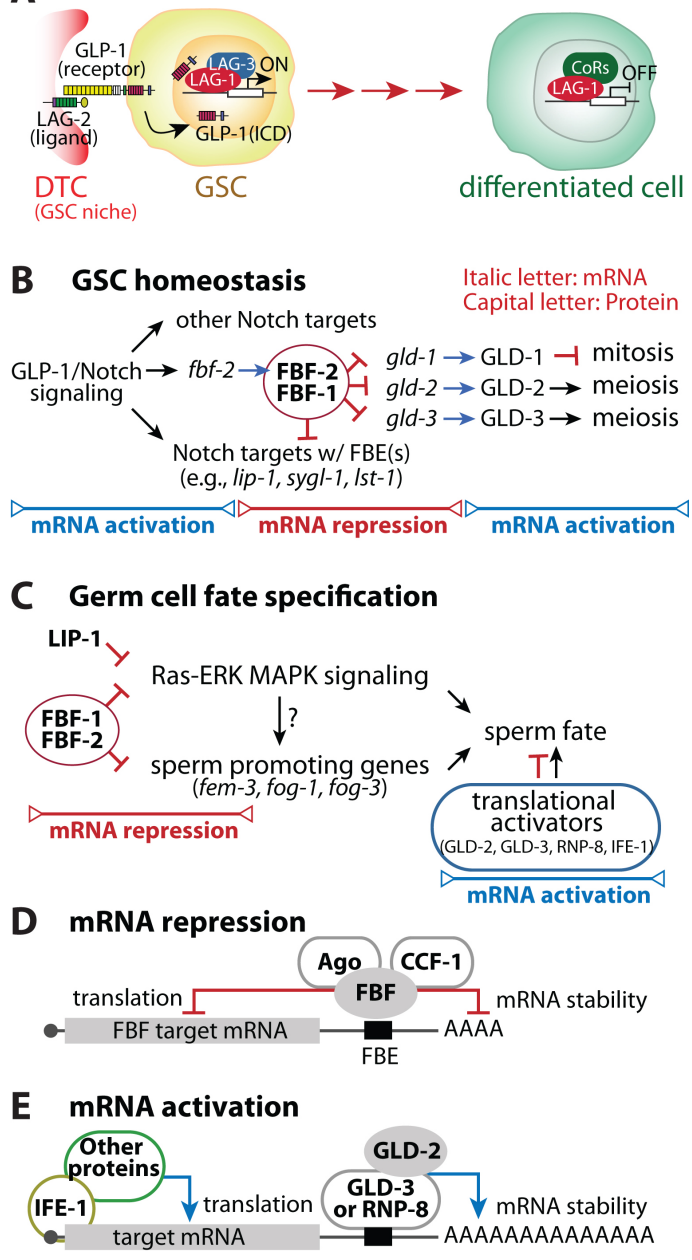

Fig. 2. A systematic RNA selection mechanism. (A) Transcriptional activation by GLP-1/Notch signaling. DTCs function a GSC niche. LAG-2, a Notch ligand, is expressed on the DTC membrane. Notch receptor, GLP-1, is expressed on the membrane of GSC and mitotically dividing germ cells. Upon Notch activation, the GLP-1 intracellular domain (ICD) is trans-located from membrane to nucleus and forms a ternary complex with transcription activators, "LAG-1 and LAG-3" to activate the expression of target genes. Once GSCs move away from a DTC, LAG-1 interacts with transcription corepressors (CoRs) to repress the expression of target genes. (B) GSC homeostasis. GLP-1/Notch signaling activates the expression of target genes, including FBF-2. FBF-2 acts as a regulatory hub for the proliferation and differentiation of GSCs. (C) Germ cell fate specification. FBF proteins inhibit the translation of the selected target mRNAs and translational activators (e.g., GLD-2, GLD-3, RNP-8, and IFE-1) promote the translation of the selected target mRNAs. (D) FBF proteins mediate mRNA repression. FBF proteins bind to the $\mathrm{FBE}(\mathrm{s})$ in target mRNAs and associate with CCF-1 or/and Ago to repress the stability or/and translation of target mRNAs. (E) GLD-2 and IFE-1-mediated mRNA activation. GLD-2 and its partners (GLD-3 or RNP-8) promote the stability of target mRNAs, as well as, IFE-1 and the translation initiation complex promote the translation of target mRNAs. Italic letters indicate mRNAs and capital letters indicate proteins.

\section{FBF-2, A REGULATORY HUB FOR GSC HOMEOSTASIS}

FBF-2 is expressed in the GSC region $(15,18)$. FBF-1 and FBF-2 (collectively known as FBF) are two, nearly identical PUF RNA-binding proteins that regulate the switch from mitosis to meiosis in the $C$. elegans germline $(7,15)$. Thus, in fbf- 1 fbf-2 double mutants, all GSCs leave the mitotic cell cycle, enter the meiotic cell cycle and undergo spermatogenesis (7). FBF proteins are translational repressors that specifically bind defined sequence element(s) in the 3'UTR (UnTranslated Region) of target mRNAs $(4,15,16,19,20)$ (Fig. 2D). FBF proteins inhibit mRNA stability and its translation by recruiting CCF-1 (Pop2P deadenylase homolog) or/and Argonaute (Ago, microRNA-binding proteins) proteins $(21,22)$ (Fig. 2D).

A recent genome-wide study identified an FBF target list of 1,350 mRNAs, using immunoprecipitation of FBF with associated mRNAs followed by microarray analysis (19). Interestingly, several FBF targets are also targets of GLP-1/Notch activated transcription $(14,16)$. These two findings suggest that GLP-1/Notch target genes might be down-regulated post-transcriptionally by FBF proteins in the GSC region.

Why are mRNAs of GLP-1/Notch target genes also repressed by FBF-2? One possible explanation is that FBF-2 maintains a balance between proliferation and differentiation (called "GSC homeostasis") by moderately suppressing both cellular states. This regulation, called "dual negative regulation", maintains cellular homeostasis $(2,23)$. Therefore, single mutations do not affect either self-renewal or differentiation of GSCs. However, when multiple genes are affected, GSC fate is altered. In addition, FBF proteins repress the translation of mRNAs that normally promote differentiation of GSCs.

The repressed mRNAs include GLD-1 (a KH-motif containing RNA-binding protein) (7), GLD-2 (a cytoplasmic poly(A) polymerase) (24), and GLD-3 (a bicaudal homolog) (25) (Fig. 2B). The GLD proteins are critical for either promoting the differentiation of GSCs or inhibiting the proliferation of GSCs. Our model proposes that FBF proteins act a central regulatory hub for GSC homeostasis (Fig. 2B).

\section{SELECTIVE mRNA REPRESSION/ACTIVATION MECHANISMS SPECIFY GERM CELL FATE}

Once GSCs enter meiosis, dynamic changes in gene expression specify the germ cell fate (4). Normally, C. elegans hermaphrodites make sperm as larvae and oocytes as adults. This fate appears to be programmed in the early meiotic region (8). Here, sperm-promoting genes (e.g., fem-3, fog- 1 , and fog-3) are expressed in the fourth larval stage (L4), but are dramatically decreased when cell fate is switched to oogenesis in young adult stages (26-28). Notably, FBF proteins promote the sperm-to-oocyte switch by inhibiting the expression of sperm-promoting genes $(19,29)$ (Fig. 2C).

We recently reported that $C$. elegans Ras-ERK MAPK signaling promotes sperm fate specification (30) (Fig. 2C). One po- 
tential target of Ras-ERK MAPK signaling is FOG-3 (a homolog of TOB/BTG anti-proliferative proteins) (26). In C. elegans germline, FOG-3 directs germ cells to adopt sperm fate at the expense of oogenesis $(26,31)$. We reported that unphosphorylated FOG-3 initiates the sperm fate program, but phosphorylated FOG-3 maintains continuous sperm production typical of males (26). Notably, FBF proteins inhibit the expression of both mpk-1 (an ERK homolog) (20) and fog-3 mRNAs $(27,32)$ (Fig. 2C). These findings suggest that FBF proteins selectively repress both sperm-promoting genes and the MPK-1/ERK MAPK signaling pathway to program sperm fate. We also found that FBF proteins promote spermatogenesis by inhibiting cell cycle regulators (Lee et al., unpublished results). Notably, male germ cells appear to have faster cell cycle progression than female germ cells (33). This finding suggests an additional role for cell cycle regulators in germ cell fate specification.

In addition to selective mRNA repression, a few RNA regulators can alternately promote the translation of their target mRNAs. For example, C. elegans GLD-2 forms an active poly(A) polymerase (PAP) when it interacts with multiple RNA-binding proteins to promote the stability and translation of target mRNAs (34) (Fig. 2E). GLD-2 controls several developmental processes, including entry into the meiotic cell cycle, and progression through both spermatogenesis and oogenesis (35-38). Two RNA-binding partners (RNP-8 and GLD-3) associated with GLD-2 have been identified $(36,37)$ (Fig. 2E). RNP-8 has an RNA recognition motif (RRM) and binds purine-rich RNA sequences (37), whereas GLD-3 belongs to the Bicaudal-C family of RNA-binding proteins (25).

The GLD-2/GLD-3 PAP activates mRNA poly(A) elongation and translation of sperm fate-promoting genes (37) (Fig. 2C and 2E). In contrast, the GLD-2/RNP-8 complex activates mRNA translation of oogenic fate-promoting genes (37) (Fig. $2 \mathrm{C}$ and $2 \mathrm{E}$ ). Mutants lacking GLD-2 are doubly defective in gametogenesis: aberrant spermatocytes occur proximally instead of mature sperm and no oocyte-like cells are observed (35).

In addition to the GLD-2-mediated polyadenylation role in gametogenesis, the translational recruitment of mRNAs mediated by $C$. elegans IFE-1 also regulate germ cell fate specification in C. elegans (39) (Fig. 2C and 2E). IFE-1 is a germline-specific isoform of translation factor elF4E, one of five isoforms of the mRNA cap-binding protein in C. elegans, uniquely associates with the germ granule (called $\mathrm{P}$ granule in $\mathrm{C}$. elegans). The germ granule shares components with the $\mathrm{P}$ bodies and stress granules in mammals (40). Interestingly, a mutant lacking IFE-1 shows a temperature sensitive arrest germ cells in secondary spermatocytes and a modest temperature-insensitive defect in oocyte development, resulting in sterility (39).

Several repressed germ cell determinant mRNAs, including gld-1 and $g / p-1$, are preferentially translated by IFE- 1 in region-specific recruitment events both in early and late germ cell differentiation steps (39). These and other studies support a model in which translational repression and selective activation of mRNAs may coordinate germ cell fate in the premeiotic region of the C. elegans germline.

\section{ABERRANT TRANSLATIONAL REGULATION AND ABNORMAL GERMLINE DEVELOPMENT}

C. elegans GSCs are established in the early larval gonad and continuously maintain their population by controlling the balance between self-renewal and differentiation. Aberrant regulation of this balance is often associated with germline tumors and infertility (3). Therefore, studying the regulatory pathways controlling the balance between these two states is critical to understand how the aberrant regulation of GSCs causes such tumors. Although we have gained significant understanding of transcriptional regulation of GSCs and cell fate in vertebrates, little is known about how translational regulators control GSC fate.

In C. elegans, many translational regulators and RNA-binding proteins are identified genetically. For example, FBF (FBF-1 and FBF-2) and GLD (GLD-1, GLD-2, and GLD-3) proteins are critical for GSC self-renewal and differentiation (4). Mutants lacking FBF proteins do not maintain GSCs and all cells differentiate into sperm (7) (Fig. 2B and 2C). As such, FBF proteins are required for GSC maintenance and oogenic fate specification. Once GSCs enter the meiotic cell cycle, the GLD-1 protein represses mitosis by inhibiting GLP-1/Notch signaling. It also represses oogenic fate by inhibiting the translation of sperm-promoting gene mRNAs (e.g., tra-2) (41).

In parallel with the function of GLD-1, GLD-2 and GLD-3 together promote polyadenylation and translation of target mRNAs (37). One of GLD-2/GLD-3 targets is g/d-1 mRNA (42). All GLD proteins promote the meiotic entry of GSCs at the translational level. Therefore, mutations in gld genes promote germline tumors, with enhanced and uncontrolled germ cell proliferation (35). These translational regulators also control germ cell fate in the premeiotic germline.

One of key translational regulators is GLD-2. GLD-2 and its partners (GLD-3 and RNP-8) control the germ cell fate (sperm or oocyte) in a combinational faction: GLD-2/GLD-3 complex drives the sperm fate and GLD-2/RNP-8 complex drives the oocyte fate (37). Notably, GLD-3 and RNP-8 antagonize each other in the sperm/oocyte decision (37). Moreover, GLD-3 also binds FBF and inhibits its repression of target mRNAs (25).

How do GSC regulators govern germ cell fate? The answer is not yet clear, but we propose that these RNA-binding proteins, polyadenylation factors, and translation initiation factors regulate the translation of target mRNAs at different places and times in the germline. Moreover, regulatory mechanisms for GSC homeostasis and cell fate specification are closely linked (8). Furthermore, GSC regulators and their target mRNAs regulate each other. These dual reciprocal regulations appear to form a spatial boundary in germ cell fate decisions (mitosis/ meiosis and sperm/oocyte). Disruption of this regulatory circuit leads to GSC loss, germline tumor, sperm/oocyte switching, or other abnormal germ cell fate, which in turn, result in infertility. 


\section{CONCLUSIONS}

In this review, we describe a new mechanism for C. elegans GSC homeostasis and their cell fate specification through several mRNA selection processes. In the GSC region, GLP-1/ Notch signaling activates the expression of target genes. FBF-2, one of C. elegans GLP-1/Notch targets, likely controls GSC homeostasis by inhibiting both the proliferation and differentiation of GSCs. Once GSCs enter pre-meiotic cell cycle, FBF-2 selectively represses its target mRNAs, associated with sperm fate specification. In addition, positive translational regulators selectively activate mRNAs, associated with oogenic fate specification. These multistep mRNA selections lead germ cells progressively to a designated cell fate, preventing abnormal development.

Interestingly, most regulators involved in this mechanism are localized to C. elegans P-granules (analogous to germ granule in mammals) $(18,43)$. C. elegans P-granules are highly enriched for RNA and RNA-binding proteins and are key centers for specialized translational control $(43,44)$. Importantly, these nematode RNA regulators are highly conserved in invertebrate and vertebrate organisms, including humans. For example, the function of the PUF RNA-binding proteins is conserved throughout many species in evolution (45). Mammalian PUF proteins (e.g., PUM1 and PUM2) bind to the Pumilio binding element (PBE) in the 3'UTR of the target mRNAs. Importantly, several PUF target mRNAs are themselves conserved among C. elegans, Drosophila, and humans (19). Mammalian PUM2 is also expressed in their embryonic stem cells (46), hematopoietic stem cells (47), and germ cells (46). It is suggested that Pum2 plays a vital "identity role" in all of these stem cells. We propose that the systematic activation/repression of discrete mRNA pools may be a conserved mechanism that broadly influences both stem cell homeostasis and cell fate specification in multicellular organisms other than C. elegans.

\section{ACKNOWLEDGEMENTS}

This work was supported in part by the Vidant Medical Center Cancer Research and Education Fund, Brody Brother's Grant, Oncology Internal Grant, and NIH (1R15GM112174-01A1) to M-H.L. The authors would like to thank Dr. Lee's lab members (especially, Eunsuk Kim) as well as Jiwoo Lee and Jiah Lee (St. Peter School, Greenville, NC, USA) for the critical reading. The authors also regret not being able to cite many important primary studies due to space limitations.

\section{REFERENCES}

1. Spradling A, Fuller MT, Braun RE and Yoshida S (2011) Germline stem cells. Cold Spring Harb Perspect Biol 3, a002642

2. Datla US, Scovill NC, Brokamp AJ, Kim E, Asch AS and Lee MH (2014) Role of PUF-8/PUF protein in stem cell control, sperm-oocyte decision and cell fate reprogramming. J Cell Physiol 10, 1306-1311

3. Kobet RA, Pan X, Zhang B, Pak SC, Asch AS and Lee MH (2014) Caenorhabditis elegans: A Model System for AntiCancer Drug Discovery and Therapeutic Target Identification. Biomol Ther (Seoul) 22, 371-383

4. Kimble J and Crittenden SL (2007) Controls of germline stem cells, entry into meiosis, and the sperm/oocyte decision in Caenorhabditis elegans. Annu Rev Cell Dev Biol 23, 405-433

5. Byrd DT and Kimble J (2009) Scratching the niche that controls Caenorhabditis elegans germline stem cells. Semin Cell Dev Biol 20, 1107-1113

6. Angelo G and Van Gilst MR (2009) Starvation protects germline stem cells and extends reproductive longevity in C. elegans. Science 326, 954-958

7. Crittenden SL, Bernstein DS, Bachorik JL et al (2002) A conserved RNA-binding protein controls germline stem cells in Caenorhabditis elegans. Nature 417, 660-663

8. Morgan CT, Noble D and Kimble J (2013) Mitosis-meiosis and sperm-oocyte fate decisions are separable regulatory events. Proc Natl Acad Sci U S A 110, 3411-3416

9. Wong MD, Jin Z and Xie T (2005) Molecular mechanisms of germline stem cell regulation. Annu Rev Genet 39, 173-195

10. Austin J and Kimble J (1989) Transcript analysis of glp-1 and lin-12, homologous genes required for cell interactions during development of C. elegans. Cell 58, 565-571

11. Yochem J and Greenwald I (1989) glp-1 and lin-12, genes implicated in distinct cell-cell interactions in C. elegans, encode similar transmembrane proteins. Cell 58, 553-563

12. Crittenden SL, Rudel D, Binder J, Evans TC and Kimble J (1997) Genes required for GLP-1 asymmetry in the early Caenorhabditis elegans embryo. Dev Biol 181, 36-46

13. Crittenden SL, Eckmann CR, Wang L, Bernstein DS, Wickens M and Kimble J (2003) Regulation of the mitosis/ meiosis decision in the Caenorhabditis elegans germline. Philos Trans R Soc Lond B Biol Sci 358, 1359-1362

14. Kershner AM, Shin H, Hansen TJ and Kimble J (2014) Discovery of two GLP-1/Notch target genes that account for the role of GLP-1/Notch signaling in stem cell maintenance. Proc Natl Acad Sci U S A 111, 3739-3744

15. Lamont LB, Crittenden SL, Bernstein D, Wickens $M$ and Kimble J (2004) FBF-1 and FBF-2 regulate the size of the mitotic region in the C. elegans germline. Dev Cell 7, 697-707

16. Lee $\mathrm{MH}$, Hook B, Lamont LB, Wickens $\mathrm{M}$ and Kimble J (2006) LIP-1 phosphatase controls the extent of germline proliferation in Caenorhabditis elegans. EMBO J 25, 88-96

17. Hajnal A and Berset T (2002) The C.elegans MAPK phosphatase LIP-1 is required for the G(2)/M meiotic arrest of developing oocytes. EMBO J 21, 4317-4326

18. Voronina E, Paix A and Seydoux G (2012) The P granule component PGL-1 promotes the localization and silencing activity of the PUF protein FBF-2 in germline stem cells. Development 139, 3732-3740

19. Kershner AM and Kimble J (2010) Genome-wide analysis of mRNA targets for Caenorhabditis elegans FBF, a conserved stem cell regulator. Proc Natl Acad Sci U S A 107, 3936-3941 
20. Lee MH, Hook B, Pan G et al (2007) Conserved regulation of MAP kinase expression by PUF RNA-binding proteins. PLoS Genet 3, e233

21. Friend $\mathrm{K}$, Campbell ZT, Cooke A, Kroll-Conner P, Wickens MP and Kimble J (2012) A conserved PUF-Ago-eEF1A complex attenuates translation elongation. Nat Struct Mol Biol 19, 176-183

22. Goldstrohm AC, Hook BA, Seay DJ and Wickens $M$ (2006) PUF proteins bind Pop2p to regulate messenger RNAs. Nat Struct Mol Biol 13, 533-539

23. Whelan JT, Hollis SE, Cha DS, Asch AS and Lee MH (2012) Post-transcriptional regulation of the Ras-ERK/ MAPK signaling pathway. J Cell Physiol 227, 1235-1241

24. Millonigg S, Minasaki R, Nousch $M$ and Eckmann CR (2014) GLD-4-mediated translational activation regulates the size of the proliferative germ cell pool in the adult C. elegans germ line. PLoS Genet 10, e1004647

25. Eckmann CR, Kraemer B, Wickens M and Kimble J (2002) GLD-3, a bicaudal-C homolog that inhibits FBF to control germline sex determination in C. elegans. Dev Cell 3, 697-710

26. Lee $\mathrm{MH}$, Kim KW, Morgan CT, Morgan DE and Kimble J (2011) Phosphorylation state of a Tob/BTG protein, FOG-3, regulates initiation and maintenance of the Caenorhabditis elegans sperm fate program. Proc Natl Acad Sci U S A $108,9125-9130$

27. Thompson BE, Bernstein DS, Bachorik JL, Petcherski AG, Wickens M and Kimble J (2005) Dose-dependent control of proliferation and sperm specification by FOG-1/CPEB. Development 132, 3471-3481

28. Arur S, Ohmachi M, Berkseth M et al (2011) MPK-1 ERK controls membrane organization in $\mathrm{C}$. elegans oogenesis via a sex-determination module. Dev Cell 20, 677-688

29. Kershner A, Crittenden SL, Friend K, Sorensen EB, Porter DF and Kimble J (2013) Germline stem cells and their regulation in the nematode Caenorhabditis elegans. Adv Exp Med Biol 786, 29-46

30. Morgan CT, Lee MH and Kimble J (2010) Chemical reprogramming of Caenorhabditis elegans germ cell fate. Nat Chem Biol 6, 102-104

31. Ellis RE and Kimble J (1995) The fog-3 gene and regulation of cell fate in the germ line of Caenorhabditis elegans. Genetics 139, 561-577

32. Snow JJ, Lee $\mathrm{MH}$, Verheyden J, Kroll-Conner PL and Kimble J (2013) C. elegans FOG-3/Tob can either promote or inhibit germline proliferation, depending on gene dosage and genetic context. Oncogene 32, 2614-2621

33. Morgan DE, Crittenden SL and Kimble J (2010) The C. elegans adult male germline: stem cells and sexual dimorphism. Dev Biol 346, 204-214

34. Suh N, Jedamzik B, Eckmann CR, Wickens M and Kimble
J (2006) The GLD-2 poly(A) polymerase activates gld-1 mRNA in the Caenorhabditis elegans germ line. Proc Natl Acad Sci U S A 103, 15108-15112

35. Kadyk LC and Kimble J (1998) Genetic regulation of entry into meiosis in Caenorhabditis elegans. Development $125,1803-1813$

36. Wang L, Eckmann CR, Kadyk LC, Wickens M and Kimble J (2002) A regulatory cytoplasmic poly(A) polymerase in Caenorhabditis elegans. Nature 419, 312-316

37. Kim KW, Nykamp K, Suh N, Bachorik JL, Wang L and Kimble J (2009) Antagonism between GLD-2 binding partners controls gamete sex. Dev Cell 16, 723-733

38. Kim KW, Wilson TL and Kimble J (2010) GLD-2/RNP-8 cytoplasmic poly(A) polymerase is a broad-spectrum regulator of the oogenesis program. Proc Natl Acad Sci U S A $107,17445-17450$

39. Henderson MA, Cronland E, Dunkelbarger S, Contreras V, Strome S and Keiper BD (2009) A germline-specific isoform of elF4E (IFE-1) is required for efficient translation of stored mRNAs and maturation of both oocytes and sperm. J Cell Sci 122, 1529-1539

40. Amiri A, Keiper BD, Kawasaki I et al (2001) An isoform of elF4E is a component of germ granules and is required for spermatogenesis in C. elegans. Development 128, 3899-3912

41. Clifford R, Lee MH, Nayak S, Ohmachi M, Giorgini $F$ and Schedl T (2000) FOG-2, a novel F-box containing protein, associates with the GLD-1 RNA binding protein and directs male sex determination in the $\mathrm{C}$. elegans hermaphrodite germline. Development 127, 5265-5276

42. Suh N, Crittenden SL, Goldstrohm A et al (2009) FBF and its dual control of gld-1 expression in the Caenorhabditis elegans germline. Genetics 181, 1249-1260

43. Updike D and Strome S (2010) P granule assembly and function in Caenorhabditis elegans germ cells. J Androl $31,53-60$

44. Sengupta MS and Boag PR (2012) Germ granules and the control of mRNA translation. IUBMB Life 64, 586-594

45. Wickens M, Bernstein DS, Kimble J and Parker R (2002) A PUF family portrait: 3'UTR regulation as a way of life. Trends Genet 18, 150-157

46. Moore FL, Jaruzelska J, Fox MS et al (2003) Human Pumilio-2 is expressed in embryonic stem cells and germ cells and interacts with DAZ (Deleted in AZoospermia) and DAZ-like proteins. Proc Natl Acad Sci U S A 100, 538-543

47. Spassov DS and Jurecic R (2003) Mouse Pum1 and Pum2 genes, members of the Pumilio family of RNA-binding proteins, show differential expression in fetal and adult hematopoietic stem cells and progenitors. Blood Cells Mol Dis 30, 55-69 